\title{
Association of Short Antenatal Corticosteroid Administration-to-Birth Intervals With Survival and Morbidity Among Very Preterm Infants Results From the EPICE Cohort
}

\begin{abstract}
Mikael Norman, MD, PhD; Aurelie Piedvache, MSc; Klaus Børch, MD, PhD; Lene Drasbek Huusom, MD, PhD; Anna-Karin Edstedt Bonamy, MD, PhD; Elizabeth A. Howell, MD, MPP; Pierre-Henri Jarreau, MD, PhD; Rolf F. Maier, MD, PhD; Ole Pryds, MD, PhD; Liis Toome, MD, PhD; Heili Varendi, MD, PhD; Tom Weber, MD, DMSc; Emilija Wilson, RN, RM; Arno Van Heijst, MD, PhD; Marina Cuttini, MD, PhD; Jan Mazela, MD, PhD; Henrique Barros, MD, PhD; Patrick Van Reempts, MD, PhD; Elizabeth S. Draper, BSc(Hons), MPhil, PhD; Jennifer Zeitlin, MA, DSc; for the Effective Perinatal Intensive Care in Europe (EPICE) Research Group
\end{abstract}

IMPORTANCE Administration-to-birth intervals of antenatal corticosteroids (ANS) vary. The significance of this variation is unclear. Specifically, to our knowledge, the shortest effective administration-to-birth interval is unknown.

OBJECTIVE To explore the associations between ANS administration-to-birth interval and survival and morbidity among very preterm infants.

DESIGN, SETTING, AND PARTICIPANTS The Effective Perinatal Intensive Care in Europe (EPICE) study, a population-based prospective cohort study, gathered data from 19 regions in 11 European countries in 2011 and 2012 on 4594 singleton infants with gestational ages between 24 and 31 weeks, without severe anomalies and unexposed to repeated courses of ANS. Data were analyzed November 2016.

EXPOSURE Time from first injection of ANS to delivery in hours and days.

MAIN OUTCOMES AND MEASURES Three outcomes were studied: in-hospital mortality; a composite of mortality or severe neonatal morbidity, defined as an intraventricular hemorrhage grade of 3 or greater, cystic periventricular leukomalacia, surgical necrotizing enterocolitis, or stage 3 or greater retinopathy of prematurity; and severe neonatal brain injury, defined as an intraventricular hemorrhage grade of 3 or greater or cystic periventricular leukomalacia.

RESULTS Of the 4594 infants included in the cohort, 2496 infants (54.3\%) were boys, and the mean (SD) gestational age was 28.5 (2.2) weeks and mean (SD) birth weight was 1213 (400) g. Mortality for the 662 infants (14.4\%) unexposed to ANS was $20.6 \%$ (136 of 661). Administration of ANS was associated with an immediate and rapid decline in mortality, reaching a plateau with more than $50 \%$ risk reduction after an administration-to-birth interval of 18 to 36 hours. A similar pattern for timing was seen for the composite mortality or morbidity outcome, whereas a significant risk reduction of severe neonatal brain injury was associated with longer administration-to-birth intervals (greater than 48 hours). For all outcomes, the risk reduction associated with ANS was transient, with increasing mortality and risk for severe neonatal brain injury associated with administration-to-birth intervals exceeding 1 week. Under the assumption of a causal relationship between timing of ANS and mortality, a simulation of ANS administered 3 hours before delivery to infants who did not receive ANS showed that their estimated decline in mortality would be $26 \%$.

CONCLUSIONS AND RELEVANCE Antenatal corticosteroids may be effective even if given only hours before delivery. Therefore, the infants of pregnant women at risk of imminent preterm delivery may benefit from its use.

JAMA Pediatr. 2017;171(7):678-686. doi:10.1001/jamapediatrics.2017.0602 Published online May 15, 2017.
Supplemental content

Author Affiliations: Author affiliations are listed at the end of this article.

Group Information: The EPICE Research Group members are listed at the end of this article.

Corresponding Author: Mikael Norman, MD, PhD, Division of Pediatrics, Department of Clinical Science, Intervention, and Technology, K78, Karolinska Institutet, SE-141 86 Stockholm, Sweden (mikael.norman@ki.se). 
A ntenatal corticosteroids (ANS) reduce morbidity and mortality in preterm infants and are recommended for women at risk of delivery before 34 weeks' gestation..$^{1-5}$ Antenatal corticosteroids have been estimated to reduce infant mortality by $31 \%$, respiratory distress syndrome by $34 \%$, intraventricular hemorrhage by $46 \%$, and necrotizing enterocolitis by $54 \% .^{4}$

While there seems to be agreement that neonatal benefits are maximized when ANS are administered 24 hours to 7 days before delivery, ${ }^{4,6-13}$ less is known about other and narrower administration-to-birth intervals. ${ }^{5}$ In particular, to our knowledge, the latest point at which ANS can be effectively used is not known. ${ }^{3}$ Given favorable pharmacokinetics ${ }^{14}$ and rapid circulatory effects, ${ }^{15-18}$ ANS may be effective even hours before birth. At the other end of the administration-to-birth interval, some ${ }^{6-11}$ but not all ${ }^{12,13}$ studies suggest declining benefits from ANS if administered more than 7 days before preterm birth. Limitations in statistical power, varying exposures, ${ }^{5,19}$ and outcomes ${ }^{6-13}$ may contribute to such diverse results and interpretations.

Because the problem of timely prediction of preterm delivery remains unresolved, ${ }^{20}$ administration of ANS will continue to occur hours, days, or weeks before delivery. Therefore, a more detailed understanding of the importance of the administration-to-birth interval of ANS for infant outcome is needed. The objective of this study was to investigate associations between different administration-to-birth intervals of ANS in hours and morbidity and mortality in very preterm infants.

\section{Methods}

The Effective Perinatal Intensive Care in Europe (EPICE) cohort study is a population-based study on the use of evidencebased practices in very preterm births with less than 32 weeks' gestation in 19 regions across 11 European countries, conducted in 2011 and 2012 (http://www.epiceproject.eu). ${ }^{21}$ Geographic and organizational diversity, feasibility, and sample size were accounted for when selecting regions. Inclusions occurred over a 12-month period except in France (6-month period). Ethics approval was obtained in each region, as required by national legislation. The European study was also approved by the French Advisory Committee on Use of Health Data in Medical Research (CCTIRS) and the French Commission for Data Protection and Liberties (CNIL). Informed parental consent (active or passive, depending on each participating country's national legislations) was obtained; in some regions, consent requirements were waived by ethics boards for parents not included in the follow-up cohort (ie, stillbirths and neonatal deaths), as data were deidentified and from routine sources.

\section{Study Population}

From a total of 10329 births, we excluded 2429 terminations of pregnancy and stillbirths, 126 infants with severe congenital anomalies, 2336 multiples, 300 infants born at 22 and 23 weeks' gestational age (GA), ${ }^{22} 362$ infants of mothers with missing data on date and time of ANS administration, and 182 infants of mothers who had received more than 1 course of ANS.

\section{Key Points}

Question What is the shortest administration-to-birth interval of antenatal corticosteroids that promotes survival and decreases neonatal morbidity in very preterm infants?

Findings In a population-based cohort of 4594 European infants born before 32 weeks' gestation, we found that in-hospital mortality was significantly reduced when antenatal corticosteroids had been administered only a few hours prior to delivery.

Meaning Encouraging administration of antenatal corticosteroids when delivery is very imminent could result in substantial survival and health gains for very preterm infants.

A repeated course was defined as an interval between ANS injections exceeding 1 week. The final sample consisted of 4594 singleton live-births at 24 to 31 weeks' GA (Figure 1). Administration of corticosteroids during pregnancy for indications other than preterm birth was not included in the study protocol.

\section{Exposure}

For each infant, information was collected on dates and times of the first ANS injection as well as the total number of injections. Questionnaires were also sent to the heads of the maternity units regularly managing 10 or more very preterm admissions annually. Most maternity units (123 of 135 [91.1\%]) responded, and protocols for ANS, including type of drug, doses, and number and intervals of injections, were collected.

We defined our exposure as time from the first injection of ANS to delivery. We classified ANS exposure in 4 categories (no ANS, first injection <24 hours, first injection between 24 hours and 7 days, and first injection $>7$ days before birth) for descriptive analyses of the characteristics of women and newborns and for comparison with other studies. ${ }^{6}$ For more detailed analyses of associations between timing of ANS and outcome, we used administration-to-birth intervals as a continuous variable. We did not include information on 1 or several doses of ANS because of high collinearity between timing of ANS and number of doses; 866 of 1111 women (77.9\%) receiving ANS less than 24 hours prior to delivery received only 1 dose.

For some women, we only had information on the date of ANS administration but not the time. This caused problems for our classifications when administration was within 2 days of delivery (204 of 1522 women [13.4\%] delivering within 2 days). For a solution, we used multiple imputations chained equations to impute these data based on all variables in the study in addition to the time of delivery, date of administration, and number of injections received. We used 100 imputed data sets. ${ }^{23}$ Results are presented using imputed data.

\section{Outcomes}

Based on the evidence of the effects of ANS on preterm infants, ${ }^{1-5}$ the outcomes selected were in-hospital mortality, a composite of mortality or severe neonatal morbidity or both, or severe neonatal brain injury. In-hospital mortality was defined as death before discharge home. Severe neonatal morbidity was defined as an intraventricular hemorrhage grade of 3 or $4,{ }^{24}$ cystic periventricular leukomalacia, ${ }^{25}$ stage 3,4 , or 5 


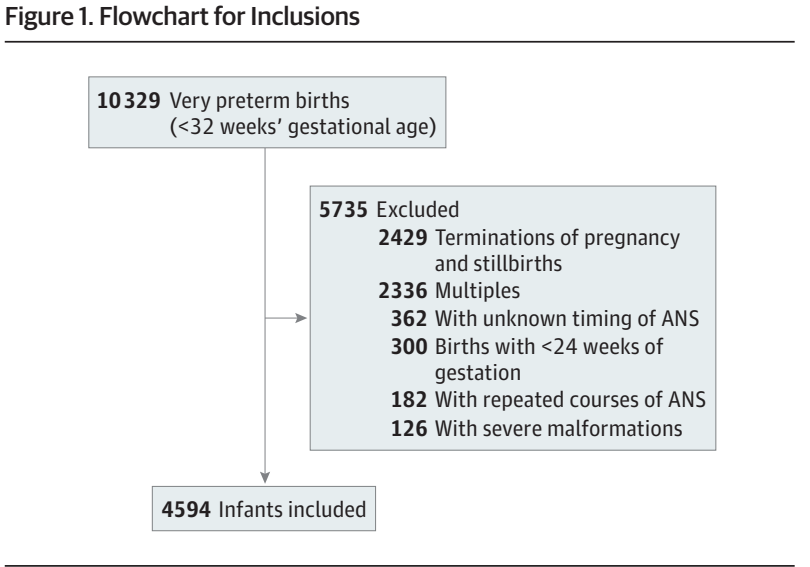

ANS indicates antenatal corticosteroids.

retinopathy of prematurity, ${ }^{26}$ or severe necrotizing enterocolitis (assessed as need for surgery or peritoneal drainage; Bell stage ${ }^{27}$ were not routinely recorded in all regions) among infants discharged alive. Severe neonatal brain injury was defined as an intraventricular hemorrhage grade of 3 or 4 , cystic periventricular leukomalacia, or both. We did not include bronchopulmonary dysplasia into our composite outcome because of regional variability in respiratory management and oxygen saturation targets have major effects on rates of bronchopulmonary dysplasia. ${ }^{28}$

\section{Covariables}

Covariables that could be potential confounders of the associations between timing of ANS and infant outcome were selected based on the literature ${ }^{4,6,29-32}$ and clinical knowledge. We included variables reflecting case mix (maternal age; parity; pregnancy complications, including preeclampsia, eclampsia, and hemolysis, elevated liver enzyme levels, low platelet count syndrome; preterm prelabor rupture of membranes; GA [defined as best assessment based on last menstrual period or antenatal ultrasonography, part of routine obstetric care in all regions]; smallness for gestational age [defined as birth weight less than the third and between the third and 10th centiles, based on customized intrauterine growth curves ${ }^{33}$ ]; and infant sex) and variables related to management of the delivery (birth at a level III neonatal unit; mode of delivery; and delivery on same day as admission to hospital). Preeclampsia was defined as hypertension after 20 weeks' GA (blood pressure of 140/90 mm Hg or higher) and proteinuria with a protein level of $0.3 \mathrm{~g} / \mathrm{L}$ or greater, and eclampsia was defined as hypertension associated with convulsions or coma. Preterm prelabor rupture of membranes was defined as spontaneous rupture of membranes 12 hours or more before onset of contractions.

\section{Statistical Analyses}

We first compared perinatal characteristics in our study population by timing of ANS administration using 4 categories. We then modeled the associations between ANS administration-to-birth intervals and the 3 outcomes using the 4-category variable and a continuous variable in hours. For the latter analysis, we used restricted cubic splines with
5 knots located at the fifth, 20th, 28th, 40th, and 95th percentiles (knots placed at $0,1,6,24$, and 520 hours) for mortality and the composite mortality and morbidity outcome. We selected 3 knots close to each other because of strong nonlinearity in the first 24 hours after ANS. For severe neonatal brain injury, we used the default percentiles (knots placed at $0,6,49,122$, and 525 hours) used by Harrell. ${ }^{34}$ Generalized linear models, assuming a Poisson distribution, were used to estimate risk ratios ${ }^{35}$ across exposures for all analyses. Adjusted models included population case mix and pregnancy management variables, as described above. Region was included as a fixed effect, and we accounted for clustering between observations within regions.

Finally, we assessed the effect on in-hospital mortality if all infants without ANS had been administered ANS less than 3 hours, 3 to 5 hours, and 6 to 12 hours before delivery by rerunning our final 4-category model and subdividing the lessthan-24-hours category into these subgroups; we then predicted mortality after setting the population with no ANS sequentially to the first, second, and third subgroups.

M.N., A.P., and J.Z. analyzed the data. Analyses were carried out using STATA version 14.0 SE (StataCorp).

\section{Results}

The most common ANS protocol was two 12-mg injections 24 hours apart (performed in 105 of 123 units [85.4\%]), followed by two 12-mg injections 12 hours apart (performed in 13 units [10.6\%]). Another 4 units (3.3\%) used other protocols. Betamethasone was used in 97 units (78.9\%), dexamethasone in 6 units (4.9\%), and both in 19 units (15.4\%) (eTable 1 in the Supplement).

\section{Distribution of Administration-to-Birth Intervals}

Fewer than half of included women (1871 of 4594 [40.7\%]) received ANS 24 hours to 7 days before delivery, ie, by current standards, the administration-to-birth interval considered to be optimal. Many women received ANS closer to delivery (1111 [24.2\%]) or did not receive ANS (662 [14.4\%]) (Table 1). The cumulative distribution of administration-to-birth intervals are presented in eFigure 1 in the Supplement. Administration and timing of ANS varied across the 19 European regions (eFigure 2 in the Supplement).

\section{Perinatal Characteristics by Timing of ANS}

Variables reflecting population case mix and management of preterm delivery differed between ANS categories, with the exception of infant sex (Table 1). In particular, women receiving ANS more than 7 days before delivery were more likely to have preterm prelabor rupture of membranes and a higher GA and to deliver in a level III unit. Of patients with missing data, 235 of 362 (64.9\%) came from 3 of the 19 regions, reflecting difficulties in data collection. After adjustment on region, infants with missing information were more often born to mothers with preeclampsia, more often delivered with cesarean section, and less often born at level III units than infants in the study population (eTable 2 in the Supplement). 


\begin{tabular}{|c|c|c|c|c|c|}
\hline \multirow[b]{2}{*}{ Characteristic } & \multicolumn{4}{|c|}{ ANS Administration-to-Birth Interval, No. (\%) } & \multirow[b]{2}{*}{$P$ Value } \\
\hline & $\begin{array}{l}\text { No ANS } \\
(n=662 \\
[14.4 \%])\end{array}$ & $\begin{array}{l}<24 \mathrm{~h} \\
(\mathrm{n}=1111 \\
[24.2 \%])\end{array}$ & $\begin{array}{l}24 \mathrm{~h}-7 \mathrm{~d} \\
(\mathrm{n}=1871 \\
[40.7 \%])\end{array}$ & $\begin{array}{l}>7 \mathrm{~d} \\
(\mathrm{n}=950 \\
[20.7 \%])\end{array}$ & \\
\hline \multicolumn{6}{|l|}{ Infant } \\
\hline Gestational age, mean (SD), wk & $28.3(2.3)$ & $28.5(2.2)$ & $28.4(2.2)$ & $28.8(1.9)$ & $<.001$ \\
\hline \multicolumn{5}{|l|}{ SGA, birth weight centiles } & \multirow{4}{*}{$<.001$} \\
\hline$<3$ & $104(15.7)$ & $204(18.4)$ & $487(26)$ & $224(23.6)$ & \\
\hline $3-10$ & $62(9.4)$ & $103(9.3)$ & $221(11.8)$ & $79(8.3)$ & \\
\hline$>10$ & 496 (74.9) & $804(72.4)$ & $1162(62.1)$ & $647(68.1)$ & \\
\hline \multicolumn{5}{|l|}{ Sex } & \multirow{3}{*}{.09} \\
\hline Male & $383(57.9)$ & $608(54.8)$ & $980(52.4)$ & $525(55.3)$ & \\
\hline Female & $279(42.2)$ & $503(45.2)$ & $890(47.6)$ & $425(44.7)$ & \\
\hline \multicolumn{5}{|l|}{ Surfactant $<2$ h after birth } & \multirow{3}{*}{$<.001$} \\
\hline No & $282(47.6)$ & $560(51.6)$ & $1134(61.9)$ & $534(58.4)$ & \\
\hline Yes & $310(52.4)$ & $524(48.4)$ & $700(38.2)$ & $381(41.6)$ & \\
\hline \multicolumn{6}{|l|}{ Maternal } \\
\hline Age, mean (SD), y & $28.9(6.4)$ & $29.6(6.2)$ & $30.4(6.1)$ & $30.5(6.2)$ & $<.001$ \\
\hline \multicolumn{5}{|l|}{ Parity } & \multirow{3}{*}{$<.001$} \\
\hline Primiparous & $330(50.7)$ & $625(56.7)$ & $1073(57.7)$ & 441 (46.9) & \\
\hline Multiparous & $321(49.3)$ & $477(43.3)$ & $787(42.3)$ & $500(53.1)$ & \\
\hline \multicolumn{5}{|l|}{ Preeclampsia/eclampsia/HELLP } & \multirow{3}{*}{$<.001$} \\
\hline No & $565(89.4)$ & 888 (80.9) & $1381(74)$ & $808(85.9)$ & \\
\hline Yes & $67(10.6)$ & $209(19.1)$ & $484(26)$ & $133(14.1)$ & \\
\hline \multicolumn{5}{|l|}{ PPROM } & \multirow{3}{*}{$<.001$} \\
\hline No & $580(91.5)$ & $972(88.4)$ & $1265(67.8)$ & $540(57.4)$ & \\
\hline Yes & $54(8.5)$ & $127(11.6)$ & $600(32.2)$ & 401 (42.6) & \\
\hline \multicolumn{5}{|l|}{ Mode of delivery onset } & \multirow{3}{*}{$<.001$} \\
\hline Spontaneous or induced & $414(63.6)$ & $662(60.1)$ & $918(49.5)$ & 507 (53.9) & \\
\hline Cesarean section & $237(36.4)$ & 439 (39.9) & $936(50.5)$ & $433(46.1)$ & \\
\hline \multicolumn{5}{|l|}{ Mode of delivery } & \multirow{3}{*}{$<.001$} \\
\hline Vaginal & $312(48.1)$ & $450(40.9)$ & $596(32.1)$ & $276(29.3)$ & \\
\hline Cesarean section & 337 (51.9) & $652(59.2)$ & $1263(67.9)$ & $667(70.7)$ & \\
\hline \multicolumn{5}{|l|}{ Delivery at level III unit ${ }^{\text {a }}$} & \multirow{3}{*}{$<.001$} \\
\hline No & $283(42.9)$ & $379(34.1)$ & $311(16.6)$ & $133(14)$ & \\
\hline Yes & $376(57.1)$ & $731(65.9)$ & $1559(83.4)$ & $817(86)$ & \\
\hline \multicolumn{5}{|l|}{ Delivery on day of admission } & \multirow{3}{*}{$<.001$} \\
\hline No & $203(32.6)$ & $605(56.3)$ & 1725 (94.9) & $802(88.2)$ & \\
\hline Yes & 419 (67.4) & $469(43.7)$ & $94(5.2)$ & 107 (11.8) & \\
\hline
\end{tabular}

Abbreviations: ANS, antenatal corticosteroids; HELLP, hemolysis, elevated liver enzyme levels, low platelet count syndrome; PPROM, preterm prelabor rupture of membranes; SGA, smallness for gestational age.

a Level III neonatal units were defined using local definitions of level of care.
Timing of ANS and Outcome

Using 4 categories of ANS timing, any ANS-irrespective of the administration-to-birth interval-was associated with lower infant mortality than no ANS, with the largest risk reduction for ANS administered 24 hours to 7 days before delivery (adjusted risk ratio, 0.5; 95\% CI, 0.4-0.6). Antenatal corticosteroids administered 24 hours to 7 days before delivery was also associated with lower risks of mortality or severe neonatal morbidity and of lower risk for severe neonatal brain injury than no ANS (Table 2).

Increasing the resolution of the analyses by expressing the risk for our 3 outcomes as a continuous function of the administration-to-birth interval, ANS less than 12 hours before birth was associated with an immediate and rapid decline in mor- tality, followed by a slower risk reduction and reaching a plateau with more than $50 \%$ risk reduction after an administrationto-birth interval of 18 to 36 hours (Figure 2). A similar pattern for timing of ANS was seen for the composite outcome of mortality or severe neonatal morbidity (eFigure 3 in the Supplement), whereas a significant risk reduction of severe neonatal brain injury was associated with longer administrationto-birth intervals (Figure 3). For all outcomes, the risk reduction associated with ANS was transient, with increasing mortality and risk for severe neonatal brain injury associated with ANS administration-to-birth intervals of 5 to 7 days or more.

In a simulation of providing ANS for the 661 infants in the sample who did not receive ANS, our model predicted a $26 \%$ decrease in mortality if these infants received ANS at 


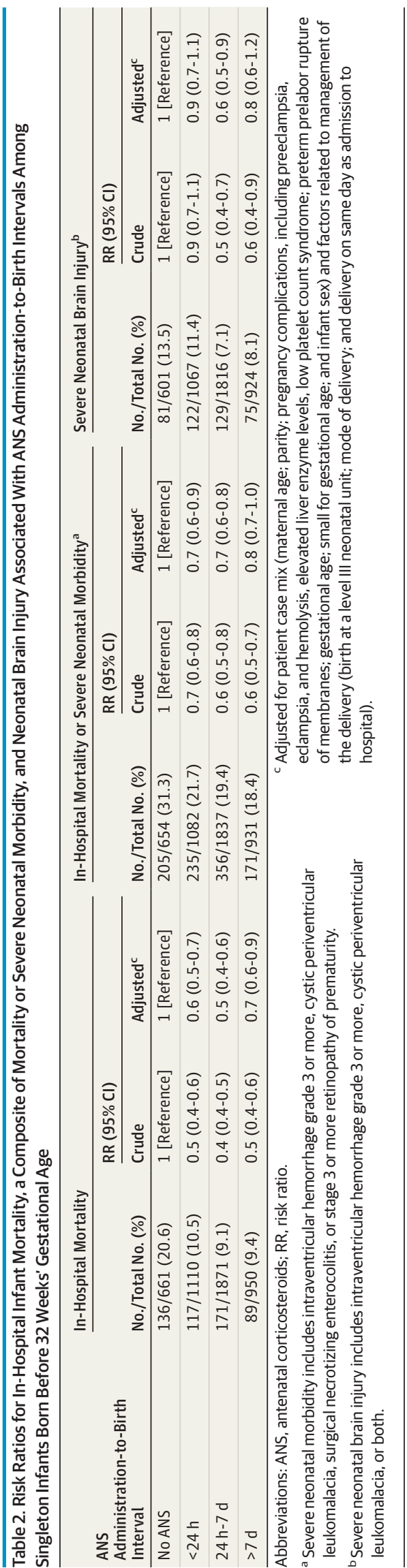

least 3 hours before delivery, a 37\% decrease if they received ANS 3 to 5 hours before delivery, and a 51\% decrease if they received ANS 6 to 12 hours before delivery (eTable 3 in the Supplement).

\section{Discussion}

Our findings suggest that infant mortality was rapidly and significantly reduced when ANS were administered only a few hours before delivery. Infant birth 12 hours following ANS administration was associated with similar mortality risk as those exposed to ANS 18 to 48 hours before delivery. Under the assumption of a causal relationship between timing of ANS and mortality, our simulations showed that if infants with no ANS prior to delivery received ANS 6 to 12 hours before delivery, their mortality was estimated to have been reduced by $51 \%$. Furthermore, the administration of ANS was associated with a significant reduction in severe neonatal morbidity.

Our findings challenge current thinking about the optimal timing of ANS. Previously, ANS administered less than 24 hours before birth have been referred to as suboptimal, ${ }^{36}$ partial, ${ }^{12}$ or incomplete, ${ }^{9}$ suggesting poorer effectiveness at administration-to-birth intervals less than 24 hours. However, the categories used in previous studies appear to have hidden clinically important effects of ANS occurring within this period. In addition, given that most (77.9\%) pregnant women in our study delivering within 24 hours of ANS only received 1 injection, significant effects may be achieved by a single ANS injection. ${ }^{19}$ In this context, we note with interest the recent statement by the National Institute for Health and Care Excellence Guideline Development Committee ${ }^{3}$; taking account of the pharmacological mechanism of action of ANS, the committee suspects that any benefits from ANS would likely be transferred even if there was only a limited amount of time between administration and time of birth.

The underlying mechanisms for the rapid actions of ANS suggested herein are not fully understood. Cord blood concentrations of ANS peak 1 hour after administration to the mother and become undetectable 2 days after the last dose. ${ }^{14}$ While induction of many corticosteroid effects involve genomic interactions and therefore take time, ${ }^{18}$ nongenomic effects of corticosteroids have in more recent years also been discovered. These effects can be very rapid and occur within minutes. ${ }^{17,18}$ Nongenomic effects may contribute to findings in preterm lambs, demonstrating beneficial corticosteroid effects on pulmonary edema and blood pressure within 8 hours after administration and significant lung function improvement within 15 hours. ${ }^{16}$ In humans, fetal movements and heart variability increased significantly within 8 hours after ANS, possibly indicating fetal well-being or fetal stress enhancing maturation. ${ }^{15}$

Although the risk reduction was slower than that associated with survival-suggesting competing outcomes (mortality and morbidity) or that the underlying mechanisms may be different-an ANS administration-to-birth interval of 48 hours to 1 week was associated with a significantly reduced risk for severe neonatal brain injury. A lower risk of severe neonatal 
Figure 2. Association Between Timing of Antenatal Corticosteroids (ANS) and In-Hospital Mortality in 4594 Very Preterm Infants

\section{A In-hospital mortality by hour}

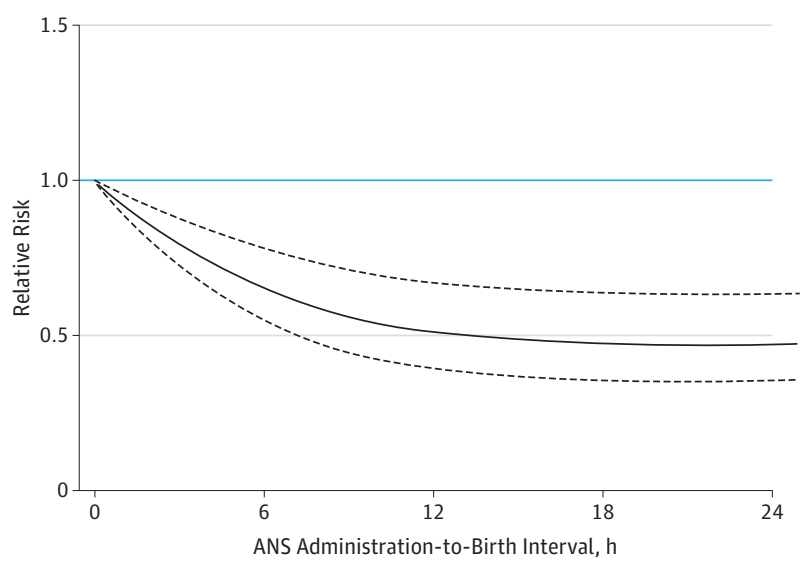

B In-hospital mortality by day

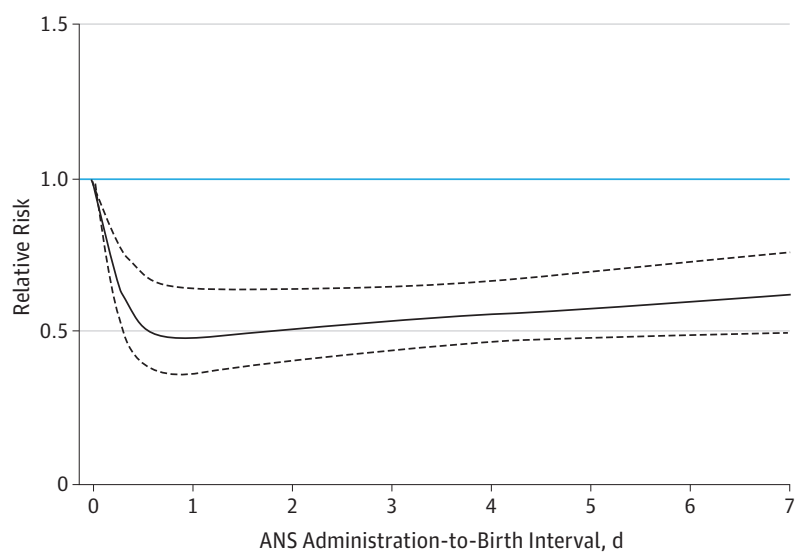

C In-hospital mortality by week

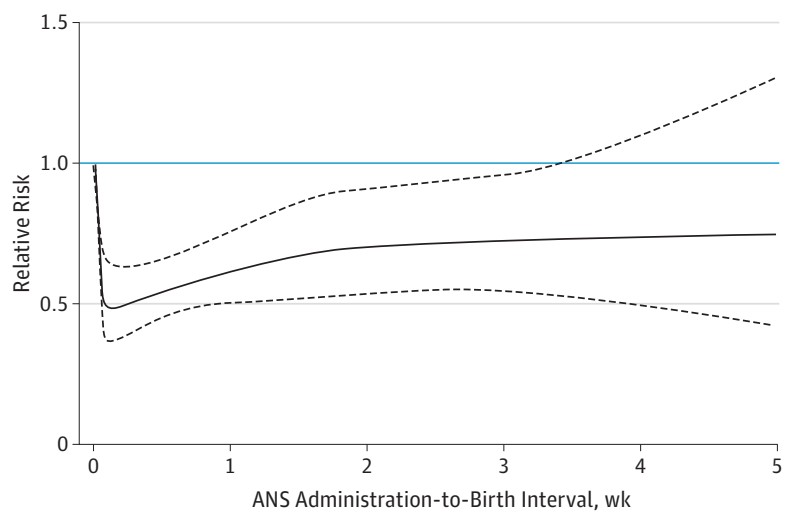

The reference relative risk represents very preterm infants who were not exposed to ANS before birth. The curves for adjusted relative risks represent means and $95 \% \mathrm{Cl}$ bands.

brain injury after ANS has previously been reported for patients at highest risk, ie, those born extremely preterm. ${ }^{22}$ A lower risk after a partial course of ANS has also been demonstrated. ${ }^{37}$ In these patients, the ANS-associated reduc-
Figure 3. Association Between Timing of Antenatal Corticosteroids (ANS) and Severe Neonatal Brain Injury in 4594 Very Preterm Infants

A IVH grade $\geq 3$ or CPVL by hour

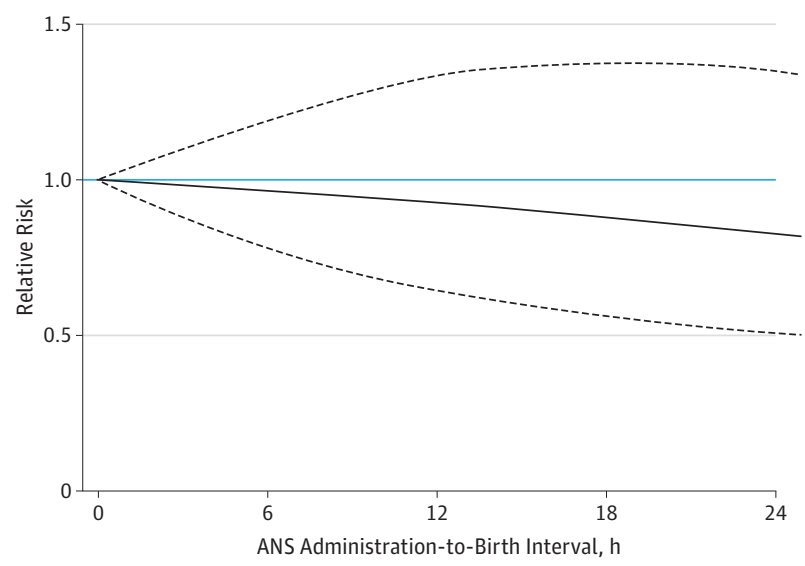

B IVH grade $\geq 3$ or $\mathrm{CPVL}$ by day

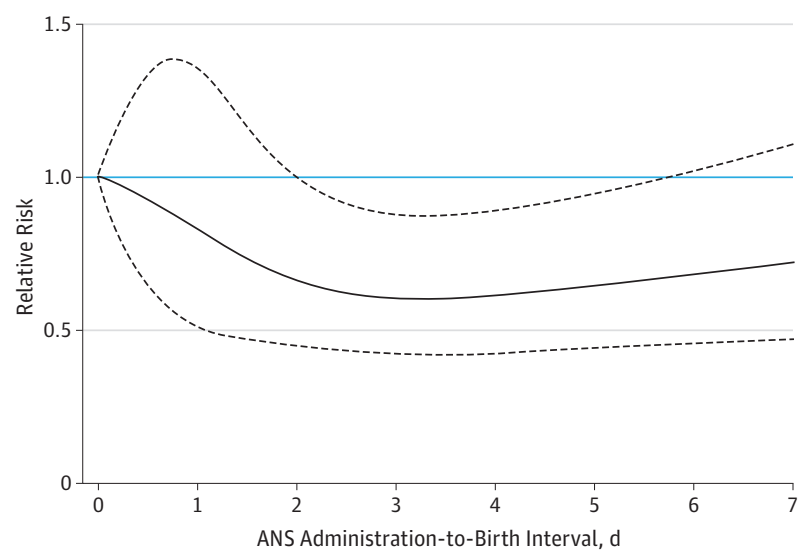

C IVH grade $\geq 3$ or CPVL by week

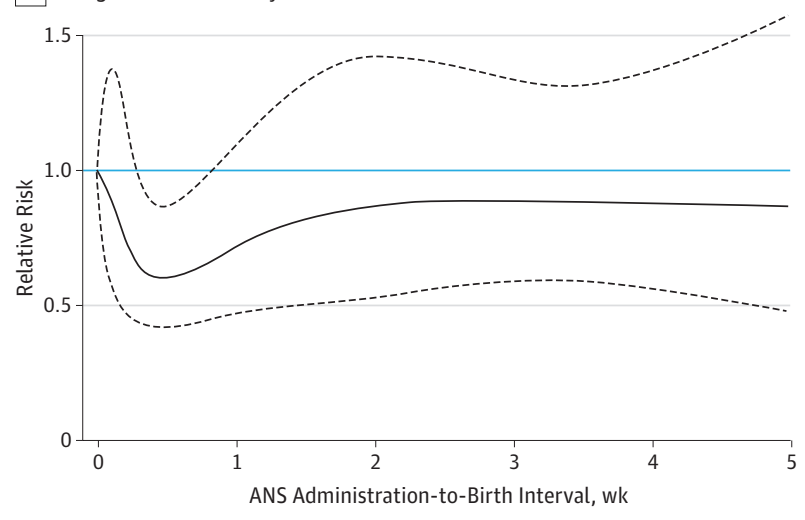

The reference relative risk represents very preterm infants who were not exposed to ANS before birth. The curves for adjusted relative risks represent means and $95 \% \mathrm{Cl}$ bands. CPVL indicates cystic periventricular leukomalacia; $\mathrm{IVH}$, intraventricular hemorrhage.

tion in severe neonatal brain injury mediated protection against later neurodevelopmental impairment. ${ }^{22,37}$

We observed a $40 \%$ increased mortality in infants with an ANS administration-to-birth interval greater than 7 days 
compared with an interval of 1 to 7 days, a group representing $18.8 \%$ of all infants in our study. In 2015, Melamed et $\mathrm{al}^{6}$ reported similar findings using the Canadian Neonatal Network. Repeated courses of corticosteroids (betamethasone was the only drug tested) have been associated with a reduction in the incidence of respiratory distress syndrome and possibly also severity of neonatal lung disease. ${ }^{38}$ At 2-year to 3-year follow-up, there was no evidence of either significant benefit or harm with a repeated course of ANS in terms of rates of neurosensory disabilities, developmental delay, or growth failure. ${ }^{2,3,38}$ Follow-up in midchildhood demonstrated long-term safety. ${ }^{39}$

\section{Strengths and Limitations}

The strengths of our study include its prospective design, large size, and heterogeneous population including both tertiary and nontertiary centers all over Europe. This ensures generalizability to a wide range of settings. Case mix, mortality, and morbidity rates are within the same ranges as previously reported from other high-income countries. ${ }^{6}$ We had power and data in sufficient detail to perform adjusted analyses on timing of ANS and outcome at very short administration-to-birth intervals. The outcomes-infant survival and major neonatal morbidities-were robust and clinically highly relevant.

Our study also has limitations. Even though we excluded infants with malformations and infants born before 24 weeks' GA, and even after adjusting for group differences presented in Table 1, residual confounding may have occurred, as the populations of women who delivered at various points after ANS administration may have been different. We investigated several outcomes, and multiple confidence intervals are reported in this article; caution should be applied when evaluating their joint statistical level. The EPICE database does not contain information on time of admission, so we could not determine admission-to-administration intervals. In addition, reasons for not treating pregnant women with ANS were not available, and we cannot exclude that withholding treatment may have occurred in some cases, especially at the lower end of GAs. However, we were able to adjust for a wide range of perinatal characteristics as well as factors reflecting management.

In our cohort, $7.0 \%$ of women received ANS but had no data on timing of administration; comparison of their characteristics suggests that these missing data may represent emergencies with suboptimal documentation. Some women had incomplete data on timing; for these patients, we used multiple imputation, which yielded results similar to complete case analysis. Our study was descriptive with no attempts in the study framework to standardize treatment, and therefore, we were unable to investigate whether one corticosteroid (or one particular dosing regimen) has advantages over another. ${ }^{5,19}$

\section{Conclusions}

In conclusion, our results suggest significant healthpromoting effects of ANS beginning just hours before delivery. This new knowledge encourages a more proactive management of women at risk for imminent preterm birth, which may help reduce infant mortality and severe neonatal brain injury. Our study also highlights that a large proportion of women remain at risk for very preterm birth more than 7 days after ANS and that their infants have increased morbidity and mortality. We suggest that future research areas should include testing optimal dosing (1 vs 2 doses) and dosing intervals of ANS. Given the suggestion of very rapid action, immediate postnatal corticosteroid rescue for very preterm infants unexposed to ANS could also be a topic for future research.

\section{ARTICLE INFORMATION}

Accepted for Publication: February 21, 2017.

Published Online: May 15, 2017.

doi:10.1001/jamapediatrics.2017.0602

Author Affiliations: Division of Pediatrics, Department of Clinical Science, Intervention, and Technology, Karolinska Institutet, Stockholm, Sweden (Norman, Wilson); Department of Neonatal Medicine, Karolinska University Hospital, Stockholm, Sweden (Norman, Wilson); INSERM Joint Research Unit 1153, Obstetrical, Perinatal, and Pediatric Epidemiology Research Team (Epopé), Center for Epidemiology and Statistics Sorbonne Paris Cité, University Hospital Department Risks in Pregnancy, Paris Descartes University, Paris, France (Piedvache, Zeitlin); Department of Neonatology, Hvidovre University Hospital, Hvidovre, Denmark (Børch, Pryds); Department of Obstetrics, Hvidovre University Hospital, Hvidovre, Denmark (Huusom, Weber); Department of Women's and Children's Health, Karolinska Institutet, Stockholm, Sweden (Bonamy); Clinical Epidemiology Unit, Department of Medicine Solna, Karolinska Institutet, Stockholm Sweden (Bonamy); Department of Population Health Science and Policy, Icahn School of Medicine at Mount Sinai, New York, New York (Howell);
Department of Obstetrics, Gynecology, and Reproductive Science, Icahn School of Medicine at Mount Sinai, New York, New York (Howell); University Paris Descartes and Department of Neonatal Medicine and Intensive Care Unit of Port-Royal, Cochin University Hospital, Assistance Publique-Hôpitaux de Paris, Paris, France (Jarreau); Children's Hospital, University Hospital, Philipp University of Marburg, Marburg, Germany (Maier); Tallinn Children's Hospital, Tallinn, Estonia (Toome); University of Tartu, Tartu, Estonia (Toome); Tartu University Hospital, University of Tartu, Tartu, Estonia (Varendi); Department of Neonatology, Radboud University Medical Center, Nijmegen, the Netherlands (Van Heijst); Research Unit of Perinatal Epidemiology, Clinical Care and Management Innovation Research Area, Bambino Gesù Children's Hospital, Rome, Italy (Cuttini); Department of Neonatology, Poznan University of Medical Sciences, Poznan, Poland (Mazela); Epidemiology Research Unit, Institute of Public Health, University of Porto, Porto, Portugal (Barros); Department of Neonatology, Antwerp University Hospital, University of Antwerp, Antwerp, Belgium (Van Reempts); Study Centre for Perinatal Epidemiology Flanders, Brussels, Belgium
(Van Reempts); Department of Health Sciences, University of Leicester, Leicester, England (Draper).

Author Contributions: Mss Piedvache and Zeitlin had full access to all of the data in the study and take responsibility for the integrity of the data and the accuracy of the data analysis.

Study concept and design: Norman, Howell, Zeitlin. Acquisition, analysis, or interpretation of data: Norman, Piedvache, Børch, Drasbek Huusom, Edstedt Bonamy, Howell, Jarreau, Maier, Pryds, Toome, Varendi, Weber, Wilson, van Heijst, Cuttini, Mazela, Van Reempts, Zeitlin.

Drafting of the manuscript: Norman, Piedvache, Zeitlin.

Critical revision of the manuscript for important intellectual content: Norman, Børch, Drasbek Huusom, Edstedt Bonamy, Howell, Jarreau, Maier, Pryds, Toome, Varendi, Weber, Wilson, van Heijst, Cuttini, Mazela, Barros, Van Reempts, Draper, Zeitlin. Statistical analysis: Norman, Piedvache, Barros, Zeitlin.

Obtained funding: Norman, Børch, Maier, Cuttini, Draper, Zeitlin.

Administrative, technical, or material support: Drasbek Huusom, Edstedt Bonamy, Maier, Pryds, Varendi, Weber, Wilson, van Heijst, Mazela, Zeitlin. Supervision: Norman, Zeitlin. 
Conflict of Interest Disclosures: None reported.

Funding/Support: The research received funding from grant agreement 259882 from the European Union Seventh Framework Program (2007-2013). Additional funding in France was provided by the French Institute of Public Health Research/Institute of Public Health and its partners, including the French Health Ministry, the National Institute of Health and Medical Research, the National Institute of Cancer, and the National Solidarity Fund for Autonomy, by grant ANR-11-EQPX-0038 from the National Research Agency through the French Equipex Program of Investments in the Future, and by the PremUp Foundation; in Poland, by 2012-2015 allocation of funds for international projects from the Polish Ministry of Science and Higher Education; and in Sweden, by regional agreement on medical training and clinical research between Stockholm County Council and Karolinska Institutet and by the Department of Neonatal Medicine at the Karolinska University Hospital.

Role of the Funder/Sponsor: The funders had no role in the design and conduct of the study; collection, management, analysis, and interpretation of the data; preparation, review, or approval of the manuscript; and decision to submit the manuscript for publication.

Group Information: In addition to the listed authors, the Effective Perinatal Intensive Care in Europe (EPICE) Research Group includes the following members: Study Centre for Perinatal Epidemiology, Brussels, Flanders, Belgium: Evelyne Martens, MSc; and Guy Martens; Department of Pediatrics, Hvidovre University Hospital, Hvidovre, Denmark: Asbjoern Hasselager, MD; INSERM Joint Research Unit 1153, Obstetrical, Perinatal, and Pediatric Epidemiology Research Team (Epopé), Center for Epidemiology and Statistics Sorbonne Paris Cité, University Hospital Department Risks in Pregnancy, Paris Descartes University, Paris, France: Pierre-Yves Ancel, MD, PhD; Béatrice Blondel, PhD; and Mercedes Bonet, MD, PhD; Division of Pediatrics 2, Bocage Hospital, INSERM CIE1, Dijon University Hospital, Dijon University, Dijon, France: Antoine Burguet, MD, PhD; Department of Neonatology, Jeanne de Flandre Hospital, Lille University Hospital, France: Patrick Truffert, MD, PhD; Institute of Quality Assurance Hesse, Eschborn, Germany: Bjoern Misselwitz, MD; Department of Obstetrics, University Hospital, Philipps University of Marburg, Marburg, Germany: Stephan Schmidt, MD, DMSc; Children's Hospital, University Hospital, University of Saarland, Saarbrücken, Germany: Ludvig Gortner, MD; Hospital Care Services, General Directorate for Health and Social Policies, Bologna, Emilia Romagna Region, Italy: Dante Baronciani, MD; Neonatal Intensive Care Unit, Department of Obstetrics and Pediatrics, Saint Maria Nuova Hospital, Florence, Italy: Giancarlo Gargano, MD; Fatebenefratelli Hospital, Rome, Italy: Rocco Agostino, MD; Agency for Public Health of Lazio Region, Health Department, Rome, Italy: Domenico DiLallo, MD; and Francesco Franco, MSc; Maternal and Child Health Institute, Marche University and Salesi Hospital, Ancona, Italy: Virgilio Carnielli, MD; Perinatal Epidemiology, Clinical Care and Management Innovation Research Area, Bambino Gesù Children's Hospital, Rome, Italy: Ileana Croci, MSc; Department of Neonatology, Wilhelmina Children's Hospital, University Medical Center Utrecht, Utrecht, the Netherlands: Corrine Koopman-Esseboom, MD, PhD; and Joppe Nijman,
MD, PhD; Department of Neonatology, Poznan University of Medical Sciences, Poznań, Poland: Janusz Gadzinowski, MD, PhD; Department of Obstetrics and Gynecology, Santa Maria University Hospital, Faculty of Medicine, University of Lisbon, Lisbon, Portugal: Luís Mendes Graça, MD, PhD; Faculty of Medicine, University of Lisbon, Lisbon Academic Medical Center, Lisbon, Portugal: Maria do Céu Machado, MD, PhD; Epidemiology Research Unit, Institute of Public Health, University of Porto Porto, Portugal: Carina Rodrigues, MSc; and Teresa Rodrigues, MD, PhD; Department of Clinical Epidemiology, Predictive Medicine, and Public Health, University of Porto Medical School, Porto, Portugal: Teresa Rodrigues, MD, PhD; Department of Gynecology and Obstetrics, Centro Hospitalar São João, Porto, Portugal: Teresa Rodrigues, MD, PhD: Department of Health Sciences, University of Leicester, Leicester, England: Elaine Boyle, MBCHB, MD, PhD; and Brad N. Manktelow, BSc, MSc, PhD; Newcastle University, Newcastle upon Tyne, England: Alan C. Fenton, MD.

\section{REFERENCES}

1. NIH Consensus Development Panel on the Effect of Corticosteroids for Fetal Maturation on Perinatal Outcomes. Effect of corticosteroids for fetal maturation on perinatal outcomes. JAMA. 1995;273 (5):413-418.

2. World Health Organization. WHO Recommendations on Interventions to Improve Preterm Birth Outcomes. Geneva, Switzerland: WHO Press; 2015.

3. National Institute for Health and Care Excellence. National Institute for Health and Care Excellence Clinical Guidelines. London, England: Preterm Labour and Birth; 2015.

4. Roberts D, Dalziel S. Antenatal corticosteroids for accelerating fetal lung maturation for women at risk of preterm birth. Cochrane Database Syst Rev. 2006;3(3):CD004454.

5. Brownfoot FC, Gagliardi DI, Bain E, Middleton P, Crowther CA. Different corticosteroids and regimens for accelerating fetal lung maturation for women at risk of preterm birth. Cochrane Database Syst Rev. 2013;(8):CD006764.

6. Melamed N, Shah J, Soraisham A, et al. Association between antenatal corticosteroid administration-to-birth interval and outcomes of preterm neonates. Obstet Gynecol. 2015;125(6): 1377-1384.

7. Kuk JY, An JJ, Cha HH, et al. Optimal time interval between a single course of antenatal corticosteroids and delivery for reduction of respiratory distress syndrome in preterm twins Am J Obstet Gynecol. 2013;209(3):256.e1-256.e7.

8. Wilms FF, Vis JY, Pattinaja DA, et al. Relationship between the time interval from antenatal corticosteroid administration until preterm birth and the occurrence of respiratory morbidity. Am J Obstet Gynecol. 2011;205(1):49.e1-49.e7.

9. Waters TP, Mercer B. Impact of timing of antenatal corticosteroid exposure on neonatal outcomes. J Matern Fetal Neonatal Med. 2009;22 (4):311-314.

10. Ring AM, Garland JS, Stafeil BR, Carr MH, Peckman GS, Pircon RA. The effect of a prolonged time interval between antenatal corticosteroid administration and delivery on outcomes in preterm neonates: a cohort study. Am J Obstet Gynecol. 2007;196(5):457.e1-457.e6.

11. Peaceman AM, Bajaj K, Kumar P, Grobman WA The interval between a single course of antenatal steroids and delivery and its association with neonatal outcomes. Am J Obstet Gynecol. 2005;193 (3, pt 2):1165-1169.

12. Sehdev HM, Abbasi S, Robertson $P$, et al. The effects of the time interval from antenatal corticosteroid exposure to delivery on neonatal outcome of very low birth weight infants. Am J Obstet Gynecol. 2004;191(4):1409-1413.

13. Vermillion ST, Soper DE, Newman RB. Is betamethasone effective longer than 7 days after treatment? Obstet Gynecol. 2001;97(4):

491-493.

14. Ballard PL, Granberg $P$, Ballard RA.

Glucocorticoid levels in maternal and cord serum after prenatal betamethasone therapy to prevent respiratory distress syndrome. J Clin Invest. 1975;56 (6):1548-1554

15. Subtil D, Tiberghien P, Devos P, et al. Immediate and delayed effects of antenatal corticosteroids on fetal heart rate: a randomized trial that compares betamethasone acetate and phosphate, betamethasone phosphate, and dexamethasone. Am J Obstet Gynecol. 2003;188(2):524-531.

16. Ikegami M, Polk $D$, Jobe A. Minimum interval from fetal betamethasone treatment to postnatal lung responses in preterm lambs. Am J Obstet Gynecol. 1996;174(5):1408-1413.

17. Hafezi-Moghadam A, Simoncini T, Yang Z, et al. Acute cardiovascular protective effects of corticosteroids are mediated by non-transcriptional activation of endothelial nitric oxide synthase. Nat Med. 2002;8(5):473-479.

18. Lösel $R$, Wehling M. Nongenomic actions of steroid hormones. Nat Rev Mol Cell Biol. 2003;4(1): 46-56.

19. Jobe $A H$, Soll RF. Choice and dose of corticosteroid for antenatal treatments. Am 」 Obstet Gynecol. 2004;190(4):878-881.

20. Whitworth MK. Recent guidance on antenatal corticosteroids in prematurity. BMJ. 2016;352:1655

21. Zeitlin J, Manktelow BN, Piedvache A, et al; EPICE Research Group. Use of evidence based practices to improve survival without severe morbidity for very preterm infants: results from the EPICE population based cohort. BMJ. 2016;354 i2976

22. Carlo WA, McDonald SA, Fanaroff $A A$, et al; Eunice Kennedy Shriver National Institute of Child Health and Human Development Neonatal Research Network. Association of antenatal corticosteroids with mortality and neurodevelopmental outcomes among infants born at 22 to 25 weeks' gestation. JAMA. 2011;306(21):2348-2358.

23. Graham JW, Olchowski AE, Gilreath TD. How many imputations are really needed? some practical clarifications of multiple imputation theory. Prev Sci. 2007;8(3):206-213.

24. Papile LA, Burstein J, Burstein R, Koffler $H$. Incidence and evolution of subependymal and intraventricular hemorrhage: a study of infants with birth weights less than 1,500 gm. J Pediatr. 1978; 92(4):529-534. 
25. de Vries LS, Eken P, Dubowitz LM. The spectrum of leukomalacia using cranial ultrasound. Behav Brain Res. 1992;49(1):1-6.

26. International Committee for the Classification of Retinopathy of Prematurity. The International Classification of Retinopathy of Prematurity revisited. Arch Ophthalmol. 2005;123(7):991-999.

27. Bell MJ, Ternberg JL, Feigin RD, et al. Neonatal necrotizing enterocolitis: therapeutic decisions based upon clinical staging. Ann Surg. 1978;187(1): $1-7$.

28. Gortner L, Misselwitz B, Milligan D, et al; members of the MOSAIC Research Group. Rates of bronchopulmonary dysplasia in very preterm neonates in Europe: results from the MOSAIC cohort. Neonatology. 2011;99(2):112-117.

29. Källén K, Serenius F, Westgren M, Maršál K; EXPRESS Group. Impact of obstetric factors on outcome of extremely preterm births in Sweden: prospective population-based observational study (EXPRESS). Acta Obstet Gynecol Scand. 2015;94 (11):1203-1214
30. Fellman V, Hellström-Westas $L$, Norman $M$, et al; EXPRESS Group. One-year survival of extremely preterm infants after active perinatal care in Sweden. JAMA. 2009;301(21):2225-2233.

31. Johansson S, Montgomery SM, Ekbom A, et al. Preterm delivery, level of care, and infant death in Sweden: a population-based study. Pediatrics. 2004;113(5):1230-1235.

32. Patterson RM. Corticosteroids for fetal maturation. JAMA. 1995:274(12):943.

33. Mikolajczyk RT, Zhang J, Betran AP, et al. A global reference for fetal-weight and birthweight percentiles. Lancet. 2011;377(9780):1855-1861.

34. Harrell FE Jr. Regression Modeling Strategies: With Applications to Linear Models, Logistic Regression, and Survival Analysis. New York, NY: Springer; 2001.

35. Zou GY, Donner A. Extension of the modified Poisson regression model to prospective studies with correlated binary data. Stat Methods Med Res. 2013;22(6):661-670.
36. Razaz N, Skoll A, Fahey J, Allen VM, Joseph KS Trends in optimal, suboptimal, and questionably appropriate receipt of antenatal corticosteroid prophylaxis. Obstet Gynecol. 2015;125(2):288-296.

37. Chawla S, Natarajan G, Shankaran S, et al; National Institute of Child Health and Human Development Neonatal Research Network. Association of neurodevelopmental outcomes and neonatal morbidities of extremely premature infants with differential exposure to antenatal steroids. JAMA Pediatr. 2016;170(12):1164-1172

38. McKinlay $\mathrm{CJ}$, Crowther $\mathrm{CA}$, Middleton $\mathrm{P}$, Harding JE. Repeat antenatal glucocorticoids for women at risk of preterm birth: a Cochrane Systematic Review. Am J Obstet Gynecol. 2012;206 (3):187-194.

39. Crowther CA, Anderson PJ, McKinlay $\mathrm{CJ}$, et al; ACTORDS Follow-up Group. Mid-childhood outcomes of repeat antenatal corticosteroids: a randomized controlled trial. Pediatrics. 2016;138 (4):e20160947. 